\title{
Understanding insomnia as systemic disease
}

\author{
Seokho Yun, Sohye Jo \\ Department of Psychiatry, Yeungnam University Hospital, Daegu, Korea
}

Received: August 8, 2021

Revised: August 28, 2021

Accepted: August 29, 2021

Corresponding author:

Seokho Yun, MD, PhD

Department of Psychiatry,

Yeungnam University Hospital, 170

Hyeonchung-ro, Nam-gu, Daegu

42415, Korea

Tel: $+82-53-620-3340$

Fax: +82-53-329-0256

E-mail:yoonseokho87@gmail.com
Sleep plays a critical role in homeostasis of the body and mind. Insomnia is a disease that causes disturbances in the initiation and maintenance of sleep. Insomnia is known to affect not only the sleep process itself but also an individual's cognitive function and emotional regulation during the daytime. It increases the risk of various neuropsychiatric diseases such as depression, anxiety disorder, and dementia. Although it might appear that insomnia only affects the nervous system, it is also a systemic disease that affects several aspects of the body, such as the cardiovascular, endocrine, and immune systems; therefore, it increases the risk of various diseases such as hypertension, diabetes mellitus, and infection. Insomnia has a wide range of effects on our bodies because sleep is a complex and active process. However, a high proportion of patients with insomnia do not seek treatment, which results in high direct and indirect costs. This is attributed to the disregard of many of the negative effects of insomnia. Therefore, we expect that understanding insomnia as a systemic disease will provide an opportunity to understand the condition better and help prevent secondary impairment due to insomnia.

Keywords: Comorbidity; Insomnia; Neurophysiology; Sleep

\section{Introduction}

Insomnia is a disease in which there is a problem with the quality or quantity of sleep. According to Diagnostic and Statistical Manual of Mental Disorders (5th edition), difficulty in initiating sleep, difficulty in maintaining sleep, and frequent awakenings or problems returning to sleep after awakenings were described as major symptoms of insomnia [1]. The prevalence of insomnia is reported to be $2.3 \%$ to $25.5 \%$ in international studies [2]. However, the 1-year incidence rate of simple insomnia symptoms is $30.7 \%$ [3]. Direct and indirect costs due to insomnia have been investigated to reach $\$ 100$ billion per year in the United States alone [4]. Past history, family history, increased arousability, poor general health, and higher bodily pain are known risk factors for insomnia [3]. Insomnia is also a major psychiatric disorder, often comorbid with various psychiatric disorders, and is also a symptom of some psychiatric disorders [5]. Because of these characteristics, insomnia is treat- ed as a neuropsychiatric disorder, and the effects of insomnia tend to be limited to the neurocognitive and affective domains. However, insomnia is known to affect physiological processes that correspond to a wide range of areas of the body and cause various physical ailments [6]. Therefore, in this review, we will examine the aspects of insomnia as a systemic disease and explain why insomnia has a wide range of effects on mental and physical health in relation to the role that sleep plays in our bodies.

\section{Insomnia and cognitive function}

The negative effects of insomnia on daytime cognitive function are well-known, and many studies have been conducted [7]. Insomnia is known to affect various cognitive domains such as memory, concentration, and attention [8].

Since sleep itself plays an important role in memory consolidation, many studies have been conducted on the effect of insomnia

Copyright (C) 2021 Yeungnam University College of Medicine

This is an Open Access article distributed under the terms of the Creative Commons Attribution Non-Commercial License (http://creativecommons.org/licenses/by-nc/4.0/) which permits unrestricted non-commercial use, distribution, and reproduction in any medium, provided the original work is properly cited. 
on memory processes [9]. Insomnia impairs the consolidation of declarative memory, and this impairment involves an increase in cortisol levels and a decrease in slow-wave sleep [10]. Moreover, insomnia affects the impairment of episodic memory, and it is known that the severity of impairment is related to the severity of insomnia symptoms [11]. In addition, several studies have reported that insomnia causes impairment of working memory $[12,13]$. However, since other studies did not confirm the change in working memory due to insomnia, additional research is needed [14].

It is also known that various elements of attention, such as sustained attention, focused attention, and shifting attention, are affected by insomnia [14]. Sustained attention, also known as vigilance, is measured by tasks that require continuous engagement over long periods of time [15]. It was confirmed that the insomnia group had lower accuracy and longer reaction time than the control group in this sustained attention task $[16,17]$. Shifting attention, which changes the focus of attention and requires flexibility, also showed differences between insomnia patients and control groups $[18,19]$. Daytime concentration problems are one of the most common complaints of insomnia [20]. The insomnia patient group showed difficulty in concentration during the daytime compared to the control group. This concentration difficulty is thought to be due to the impairment of sustained attention and shifting attention [8].

Executive function is a set of neurocognitive skills involved in problem-solving, including working memory, inhibitory control, and shifting/flexibility. Therefore, executive function is a cognitive domain that includes some concepts, such as working memory and shifting attention previously introduced [21]. The association between executive function and insomnia has recently become an apostate issue compared to other cognitive domains, and the results tend to be inconsistent; therefore, a meta-analysis has been conducted with various studies recently. Guo et al. [22] confirmed that naming, immediate memory, delayed recall, and overall Montreal Cognitive Assessment (MoCA) scores were lower in the insomnia group using the Pittsburgh Sleep Quality Index and MoCA. They also found that an increase in the severity of insomnia translates to a more extensive and severe cognitive impairment. Fortier-Brochu and Morin [23] found that there was an impairment of episodic memory and attention in the insomnia group, which was associated with sleep continuity, sleep microstructure, and dysfunctional belief. Fulda and Schulz [24] confirmed that there was impairment in episodic memory, problem-solving, manipulation in working memory, and retention in working memory through a meta-analysis of 24 insomnia studies, but the degree of impairment remained small to moderate. They, in a meta-analysis of 18 insomnia studies, found no consistent evidence of cognitive dysfunction associated with insomnia. They noted that the difference in sample size and situational factors may have an effect as the sample size and situational factors produce different results in different studies. As such, impairment of executive function due to insomnia tends to have different results even in a meta-analysis; therefore, further evaluation is necessary.

\section{Insomnia and emotion regulation}

Sleep and emotion have bidirectional interactions with each other [25]. Therefore, chronic insomnia is known to affect emotion regulation [26]. Several studies have reported that sleep deprivation due to insomnia affects emotional valence; hence, poor sleep quality induces low positive emotions and high negative emotions, and this tendency is reported to be stronger in women [27]. However, in another study, this effect on sex was not confirmed [28]. Emotional disturbances caused by sleep deprivation are known to involve the amygdala and the medial prefrontal cortex [29]. It is also known that insomnia, negative emotions, and intrusive or unwanted thoughts tend to aggravate symptoms by composing a vicious cycle with each other [30]. A study by Markarian et al. [31] found that emotional dysregulation difficulties were associated with depression, anxiety, and stress and that sleep quality plays an important role. Another study found that aggression and impulsivity are associated with worse sleep quality and higher insomnia scores [32]. It has been reported that emotional dysregulation due to insomnia is associated with changes in heart rate variability (HRV). These results suggest that the mechanism by which insomnia causes emotional dysregulation is closely related to brain-body interactions known to reflect HRV [33]. Interoception is a concept that includes nociception, thermoception, and visceral sensation and is known to play an important role in brain-body interactions. Interoception was also confirmed to have profound effects on insomnia [34]. Heartbeat evoked potential (HEP) is a heartbeat-induced change in the electroencephalogram (EEG) signal that is known to directly reflect interoceptive processing. Changes in HEP have been reported in insomnia patients, suggesting that insomnia may be closely related to changes in interoception. [35]. In addition, in recent studies, interoception has been shown to play an important role not only in emotion regulation but also in anxiety and depression induced by sleep deprivation [36]. Recent discoveries on interoception have given strength to the hypothesis that changes in interoception due to insomnia are a key mechanism in the development of symptoms such as emotion regulation disturbance, anxiety, and depression [37]. 


\section{Insomnia and neuropsychiatric disorders}

Insomnia not only affects cognitive function and emotion regulation but is also strongly associated with several related neuropsychiatric disorders [38]. Depression and insomnia have a bidirectional relationship [39]. In a 40-year longitudinal follow-up study of medical students, it was found that individuals with baseline insomnia were twice as likely to develop the major depressive disorder than those who did not [40]. In addition, the odds ratio that an insomnia patient without depression would later develop depression was 6.2, and the odds ratio that a depressed patient without insomnia would later develop insomnia was 6.7 [41]. Insomnia is also known to increase the risk of suicidal ideation in subjects without mental disorders $[42,43]$.

Insomnia is known to increase the risk of various anxiety disorders and depression. In a population-based study of 2,393 random individuals, symptoms of insomnia related to anxiety disorders were identified as short sleep duration, daytime sleepiness, and sleep disturbance [41]. Among anxiety disorders, generalized anxiety disorder is a disease closely related to insomnia as there are sleep disturbance symptoms in the main symptoms [1]. In individuals reporting symptoms of insomnia in a French population study, generalized anxiety disorder was the most prevalent psychiatric disorder [44]. Various anxiety disorders, such as panic disorder, specific phobia, and social anxiety disorder, have been reported to be comorbid with insomnia [41].

The cross-sectional relationship between dementia and insomnia has been well-known for a long time, but recently, various cohort studies and meta-analysis studies have reported that insomnia increases the risk of all types of dementia [45]. In a nationwide population-based study in Taiwan, insomnia was found to increase the risk of Alzheimer dementia, especially if insomnia started before the age of 40 years [46]. There are several possible mechanisms by which chronic insomnia increases the risk of dementia. The hyperarousal state caused by insomnia induces overactivation of the hypothalamic-pituitary-adrenal cascade, leading to neurodegeneration [47]. In addition, aggregation of $\beta$-amyloid in the brain is considered the main cause of Alzheimer dementia. $\beta$-amyloid is actively excreted during sleep, and a decrease in $\beta$-amyloid clearance due to insomnia is also thought to increase the risk of dementia [47]. However, there is controversy regarding the amyloid hypothesis as a pathophysiology of dementia. Therefore, additional research is needed to elucidate the exact reason why insomnia increases the risk of dementia.

\section{Insomnia and physiological process}

Insomnia is also known to cause various physiological changes during the day and night. Monroe [48] found elevated rectal temperature, heart rate, basal skin resistance, and phasic vasoconstriction in poor sleepers. It is known that these changes in the autonomic nervous system are caused by an imbalance between the sympathetic and parasympathetic nervous systems [49]. Imbalance of the autonomic nervous system, which is the main pathogenesis of insomnia, is also a key mechanism in the hyperarousal model, the main pathogenesis of insomnia [50].

Since various hormones, such as growth hormone and melatonin, are involved in sleep regulation, sleep and hormones have a reciprocal relationship [51]. Therefore, insomnia causes dysregulation of various endocrine systems [52]. An increase in cortisol hormone and a change in the 24-hour metabolic rate have been observed in patients with chronic insomnia $[13,53]$. In addition, it was confirmed that sleep deprivation decreased insulin sensitivity, while insulin resistance and secretion increased, especially in elderly patients with insomnia $[54,55]$. Insomnia is also known to induce changes in the endocrine system that controls appetite. Limited total sleep time was associated with higher ghrelin and reduced leptin levels, according to the Wisconsin Sleep Cohort Study [56]. In a study by Motivala et al. [57], a study on chronic insomnia confirmed a decrease in nocturnal ghrelin, but leptin did not show a significant difference in the control group. Based on these results, insomnia is thought to induce inappropriate overeating and weight gain. Melatonin, a hormone that controls circadian rhythm, is also closely related to insomnia. Various studies have confirmed that melatonin levels are decreased in patients with insomnia. In particular, the longer the insomnia period, the greater the decrease in melatonin [58].

The immune system is also reciprocally related to sleep, and sleep regulation is regulated by the immune system. It is also established that sleep deprivation affects the immune system $[59,60]$. During sleep, cytokines such as interleukin 1 and tumor necrosis factor are known to be involved in non-rapid eye movement (NREM) sleep. Sleep deprivation is known to cause changes in immune cell number, immune function, and cytokines, as well as to make them more susceptible to viral and bacterial infections [61]. In addition, chronic insomnia has been reported to increase inflammation and mortality rates [62].

Insomnia induces various neurophysiological changes. In a meta-analysis that analyzed 23 studies using polysomnography in insomnia patients, it confirmed the changes in various sleep variables, including sleep efficiency index, total sleep time, rate of slowwave sleep, rate of rapid eye movement (REM) sleep, and rate of 
change in the wake time [63]. In addition, these neurophysiological changes were observed during the daytime. High-beta power was observed in the resting state EEG of an insomnia patient, and a change in the P200 component was reported in the event-related potential study $[64,65]$. These daytime neurophysiological changes are known to reflect the hyperarousal state caused by insomnia.

\section{Insomnia and physical disorders}

Insomnia is known to cause temporary changes in various physiological functions as well as various physical diseases [66]. Since insomnia is accompanied by changes in heart rate and blood pressure, several studies have been conducted in the past to determine whether insomnia is related to cardiovascular diseases such as hypertension, coronary heart disease, and heart failure; however, each study revealed inconsistent results [67]. However, several meta-analysis studies and nationwide population-based studies conducted over the past decade have reported that insomnia increases the risk of cardiovascular disease [67-69]. Sofi et al. [70] concluded in a meta-analysis that insomnia was associated with the development of cardiovascular disease and cardiovascular disease mortality. The onset of cardiovascular disease is known to be associated with dysregulation of the autonomic nervous system due to insomnia and metabolic syndrome due to hormonal dysregulation [71].

Insomnia is also known to cause endocrine disorders. In the case of type 2 diabetes mellitus, several studies have slightly different results, but several studies have suggested that insomnia and type 2 diabetes mellitus are related, and that insomnia may act as a risk factor for type 2 diabetes mellitus [72,73]. Although it is wellknown that abnormal thyroid function induces insomnia, the effect of insomnia on thyroid function has not been well studied [66]. However, elevated levels of thyrotropin-releasing hormone and thyroid-stimulating hormone have been reported in patients with insomnia accompanied by depression [74]. Obesity has also been reported to increase the risk of insomnia, and changes in appetite-regulating hormones, such as leptin and ghrelin, are known to be involved. However, a recently reported meta-analysis confirmed that insomnia did not directly increase the risk of obesity $[75,76]$. Therefore, further evaluation is needed to determine whether insomnia increases the risk of obesity.

Insomnia is known to increase the risk of infection and cancer because of the close relationship between sleep and the immune system [38]. A cohort study confirmed that insomnia increases the risk of respiratory tract infection, and it has been reported that patients receiving chemotherapy are more susceptible to infection if they complain of insomnia [77,78]. Many researchers have long believed that insomnia increases the risk of cancer [79]. This is not just a belief; some studies have reported that insomnia increases the risk of breast cancer [80,81]. In addition, according to a meta-analysis of cohort studies on insomnia, insomnia increases the risk of all cancers overall, but only thyroid cancer reported a significant increase in risk due to insomnia in specific cancer types [82].

\section{Insomnia as systemic disease}

We looked at examples in previous chapters where insomnia affects various systems of the body as well as the neuropsychiatric domain. Insomnia is characterized as a systemic disease as the role of sleep is not only limited to rest and conservation of energy. Moreover, sleep has an active, complex, and unique function in the body. Sleep can be divided into REM and NREM stages, and it is known that the two stages play different roles [83]. NREM stage is known as the most restorative stage, and it is known to play an important role in energy conservation and maintenance of physical health, such as hormonal regulation, $\beta$-amyloid clearance, and adaptive immune response [84]. REM sleep is a stage clearly distinguished from NREM sleep in neurophysiological characteristics, and it is known that eye movement, voluntary muscle paralysis and dreaming appear [83]. REM sleep is related to the process of emotional memory. Also, in REM sleep, muscle tone is actively suppressed, and autonomic and respiratory activation appear [85].

As such, sleep is a very dynamic process in which stages have different purposes and appear alternately, and each stage has a bidirectional relationship with different biological systems. Therefore, if this complex process is disturbed due to insomnia, it inevitably has a systemic effect on the body and mental health.

Furthermore, insomnia has a systemic effect and is not limited to a specific area as it affects distributed systems throughout the body. An example is a decrease in immune function due to insomnia. Chronic insomnia increases inflammation in the body, making it vulnerable to infection. The hyperarousal model, which is one of the main causes of insomnia, also affects the autonomic nervous system distributed in all parts of the body, so it affects not only the central nervous system but also the peripheral nervous system and various organs throughout the body. In addition, the endocrine and circulatory systems are also greatly affected.

Moreover, the systemic effect of insomnia is not limited to the body but is known to directly or indirectly affect social and occupational functions $[86,87]$. The array of systemic effects and comorbidities caused by insomnia is known to be directly related to a reduced quality of life for patients across a range of different domains [88]. Although the treatment of insomnia is important due to its 
systemic effect, it has been reported that many subjects with insomnia symptoms do not feel the need for treatment or do not receive appropriate treatment [89]. The rationale many patients neglect insomnia even though they are symptomatic is because its negative effects are undermined and its systemic sequelae are not well-known [90]. Therefore, we expect that this review, which studied the characteristics of insomnia as a systemic disease, will help reduce the enormous personal and social burden of untreated insomnia and prevent insomnia-induced secondary diseases.

\section{Conclusion}

Insomnia not only causes problems related to sleep initiation and maintenance but also has a secondary global sequela on both the mind and body. Insomnia causes functional impairment in various cognitive domains and causes emotional dysregulation. Chronic insomnia also increases the risk of various diseases, such as major depressive disorder and Alzheimer dementia. However, insomnia is not limited to these neuropsychiatric areas, but it affects various physiological systems such as the immune system, endocrine system, and circulatory system and increases the risk of various diseases such as diabetes mellitus, hypertension, cancer, and infection. Insomnia is considered a systemic disease because the sleep process is a very complex, dynamic, and globally biological phenomenon. However, despite the negative effects of insomnia, the proportion of patients who do not receive optimal treatment is very high. This is due to the very limited view of the negative effects of insomnia on our bodies. Therefore, recognizing insomnia as a systemic disease is expected to help prevent secondary impairment and reduce costs through early detection and appropriate treatment.

\section{Notes}

\section{Conflicts of interest}

No potential conflict of interest relevant to this article was reported.

\section{Author contributions}

Data curation: SJ; Project administration: SY; Writing - original draft: SJ, SY; Writing - review \& editing: SJ.

\section{ORCID}

Seokho Yun, https://orcid.org/0000-0002-2200-3494

Sohye Jo, https://orcid.org/0000-0001-9471-0713

\section{References}

1. American Psychiatric Association (APA). Diagnostic and statistical manual of mental disorders (DSM-5). 5th ed. Arlington, VA: APA; 2013.

2. Aernout E, Benradia I, Hazo JB, Sy A, Askevis-Leherpeux F, Sebbane D, et al. International study of the prevalence and factors associated with insomnia in the general population. Sleep Med 2021;82:186-92.

3. LeBlanc M, Mérette C, Savard J, Ivers H, Baillargeon L, Morin $\mathrm{CM}$. Incidence and risk factors of insomnia in a population-based sample. Sleep 2009;32:1027-37.

4. Taddei-Allen P. Economic burden and managed care considerations for the treatment of insomnia. Am J Manag Care 2020; 26(4 Suppl):S91-6.

5. Harvey AG. Insomnia: symptom or diagnosis? Clin Psychol Rev 2001;21:1037-59.

6. Fernandez-Mendoza J, Vgontzas AN. Insomnia and its impact on physical and mental health. Curr Psychiatry Rep 2013;15: 418.

7. Fortier-Brochu E, Beaulieu-Bonneau S, Ivers H, Morin CM. Insomnia and daytime cognitive performance: a meta-analysis. Sleep Med Rev 2012;16:83-94.

8. Brownlow JA, Miller KE, Gehrman PR. Insomnia and cognitive performance. Sleep Med Clin 2020;15:71-6.

9. Nissen C, Kloepfer C, Nofzinger EA, Feige B, Voderholzer U, Riemann D. Impaired sleep-related memory consolidation in primary insomnia: a pilot study. Sleep 2006;29:1068-73.

10. Backhaus J, Junghanns K, Born J, Hohaus K, Faasch F, Hohagen F. Impaired declarative memory consolidation during sleep in patients with primary insomnia: influence of sleep architecture and nocturnal cortisol release. Biol Psychiatry 2006;60:132430.

11. Drummond SP, Walker M, Almklov E, Campos M, Anderson DE, Straus LD. Neural correlates of working memory performance in primary insomnia. Sleep 2013;36:1307-16.

12. Vignola A, Lamoureux C, Bastien CH, Morin CM. Effects of chronic insomnia and use of benzodiazepines on daytime performance in older adults.J Gerontol B Psychol Sci Soc Sci 2000; 55:P54-62.

13. Bonnet MH, Arand DL. 24-Hour metabolic rate in insomniacs and matched normal sleepers. Sleep 1995;18:581-8.

14. Shekleton JA, Rogers NL, Rajaratnam SM. Searching for the daytime impairments of primary insomnia. Sleep Med Rev 2010;14:47-60.

15. Shallice T, Stuss DT, Alexander MP, Picton TW, Derkzen D. The multiple dimensions of sustained attention. Cortex 2008; 
44:794-805.

16. Hauri PJ. Cognitive deficits in insomnia patients. Acta Neurol Belg 1997;97:113-7.

17. Altena E, Van Der Werf YD, Strijers RL, Van Someren EJ. Sleep loss affects vigilance: effects of chronic insomnia and sleep therapy.J Sleep Res 2008; 17:335-43.

18. Edinger JD, Means MK, Carney CE, Krystal AD. Psychomotor performance deficits and their relation to prior nights' sleep among individuals with primary insomnia. Sleep 2008;31:599607.

19. Edinger JD, Glenn DM, Bastian LA, Marsh GR. Slow-wave sleep and waking cognitive performance II: findings among middle-aged adults with and without insomnia complaints. Physiol Behav 2000;70:127-34.

20. Roth T, Ancoli-Israel S. Daytime consequences and correlates of insomnia in the United States: results of the 1991 National Sleep Foundation Survey. II. Sleep 1999;22(Suppl 2):S354-8.

21. Vasisht KP, Kessler LE, Booth JN 3rd, Imperial JG, Penev PD. Differences in insulin secretion and sensitivity in short-sleep insomnia. Sleep 2013;36:955-7.

22. Guo H, Wei M, Ding W. Changes in cognitive function in patients with primary insomnia. Shanghai Arch Psychiatry 2017; 29:137-45.

23. Fortier-Brochu E, Morin CM. Cognitive impairment in individuals with insomnia: clinical significance and correlates. Sleep 2014;37:1787-98.

24. Fulda S, Schulz H. Cognitive dysfunction in sleep disorders. Sleep Med Rev 2001;5:423-45.

25. Gruber R, CassoffJ. The interplay between sleep and emotion regulation: conceptual framework empirical evidence and future directions. Curr Psychiatry Rep 2014;16:500.

26. Kyle SD, Beattie L, Spiegelhalder K, Rogers Z, Espie CA. Altered emotion perception in insomnia disorder. Sleep 2014; 37:775-83.

27. McCrae CS, McNamara JP, Rowe MA, Dzierzewski JM, Dirk J, Marsiske M, et al. Sleep and affect in older adults: using multilevel modeling to examine daily associations. J Sleep Res 2008; 17:42-53.

28. Scott BA, Judge TA. Insomnia, emotions, and job satisfaction: a multilevel study. J Manag 2006;32:622-45.

29. Huang Z, Liang P, Jia X, Zhan S, Li N, Ding Y, et al. Abnormal amygdala connectivity in patients with primary insomnia: evidence from resting state fMRI. Eur J Radiol 2012;81:1288-95.

30. Harvey AG. I can't sleep, my mind is racing! An investigation of strategies of thought control in insomnia. Behav Cogn Psychother 2001;29:3-11.

31. Markarian SA, Pickett SM, Deveson DF, Kanona BB. A model of BIS/BAS sensitivity, emotion regulation difficulties, and depression, anxiety, and stress symptoms in relation to sleep quality. Psychiatry Res 2013;210:281-6.

32. Kamphuis J, Dijk DJ, Spreen M, Lancel M. The relation between poor sleep, impulsivity and aggression in forensic psychiatric patients. Physiol Behav 2014;123:168-73.

33. Ingjaldsson JT, Laberg JC, Thayer JF. Reduced heart rate variability in chronic alcohol abuse: relationship with negative mood, chronic thought suppression, and compulsive drinking. Biol Psychiatry 2003;54:1427-36.

34. Craig AD. Interoception: the sense of the physiological condition of the body. Curr Opin Neurobiol 2003;13:500-5.

35. Wei Y, Ramautar JR, Colombo MA, Stoffers D, Gómez-Herrero $\mathrm{G}$, van der Meijden WP, et al. I keep a close watch on this heart of mine: increased interoception in insomnia. Sleep 2016;39: 2113-24.

36. Paulus MP, Stein MB. Interoception in anxiety and depression. Brain Struct Funct 2010;214:451-63.

37. Stephan KE, Manjaly ZM, Mathys CD, Weber LA, Paliwal S, Gard T, et al. Allostatic self-efficacy: a metacognitive theory of dyshomeostasis-induced fatigue and depression. Front Hum Neurosci 2016;10:550.

38. Taylor DJ, Mallory LJ, Lichstein KL, Durrence HH, Riedel BW, Bush AJ. Comorbidity of chronic insomnia with medical problems. Sleep 2007;30:213-8.

39. Ettensohn M, Soto Y, Bassi B, Khurshid KA. Sleep problems and disorders in patients with depression. Psychiatr Ann 2016; 46:390-5.

40. Chang PP, Ford DE, Mead LA, Cooper-Patrick L, Klag MJ. Insomnia in young men and subsequent depression: the Johns Hopkins Precursors Study. Am J Epidemiol 1997;146:105-14.

41. Khurshid KA. Comorbid insomnia and psychiatric disorders: an update. Innov Clin Neurosci 2018;15:28-32.

42. Thase ME. Depression, sleep, and antidepressants. J Clin Psychiatry 1998;59(Suppl 4):55-65.

43. Franzen PL, Buysse DJ. Sleep disturbances and depression: risk relationships for subsequent depression and therapeutic implications. Dialogues Clin Neurosci 2008;10:473-81.

44. Ohayon MM. Prevalence of DSM-IV diagnostic criteria of insomnia: distinguishing insomnia related to mental disorders from sleep disorders. J Psychiatr Res 1997;31:333-46.

45. de Almondes KM, Costa MV, Malloy-Diniz LF, Diniz BS. Insomnia and risk of dementia in older adults: systematic review and meta-analysis. J Psychiatr Res 2016;77:109-15.

46. Chen PL, Lee WJ, Sun WZ, Oyang YJ, Fuh JL. Risk of dementia in patients with insomnia and long-term use of hypnotics: a population-based retrospective cohort study. PLoS One 2012; 
7:e49113.

47. Bombois S, Derambure P, Pasquier F, Monaca C. Sleep disorders in aging and dementia. J Nutr Health Aging 2010; 14:2127.

48. Monroe LJ. Psychological and physiological differences between good and poor sleepers. J Abnorm Psychol 1967;72: 255-64.

49. Roth T, Roehrs T. Insomnia: epidemiology, characteristics, and consequences. Clin Cornerstone 2003;5:5-15.

50. Levenson JC, Kay DB, Buysse DJ. The pathophysiology of insomnia. Chest 2015;147:1179-92.

51. Morris CJ, Aeschbach D, Scheer FA. Circadian system, sleep and endocrinology. Mol Cell Endocrinol 2012;349:91-104.

52. Morgan D, Tsai SC. Sleep and the endocrine system. Crit Care Clin 2015;31:403-18.

53. Vgontzas AN, Bixler EO, Lin HM, Prolo P, Mastorakos G, Vela-Bueno A, et al. Chronic insomnia is associated with nyctohemeral activation of the hypothalamic-pituitary-adrenal axis: clinical implications. J Clin Endocrinol Metab 2001;86:378794.

54. Buxton OM, Pavlova M, Reid EW, Wang W, Simonson DC, Adler GK. Sleep restriction for 1 week reduces insulin sensitivity in healthy men. Diabetes 2010;59:2126-33.

55. Yamamoto N, Yamanaka G, Ishizawa K, Ishikawa M, Murakami $\mathrm{S}$, Yamanaka T, et al. Insomnia increases insulin resistance and insulin secretion in elderly people. J Am Geriatr Soc 2010;58: 801-4.

56. Taheri S, Lin L, Austin D, Young T, Mignot E. Short sleep duration is associated with reduced leptin, elevated ghrelin, and increased body mass index. PLoS Med 2004;1:e62.

57. Motivala SJ, Tomiyama AJ, Ziegler M, Khandrika S, Irwin MR. Nocturnal levels of ghrelin and leptin and sleep in chronic insomnia. Psychoneuroendocrinology 2009;34:540-5.

58. Hajak G, Rodenbeck A, Staedt J, Bandelow B, Huether G, Rüther E. Nocturnal plasma melatonin levels in patients suffering from chronic primary insomnia. J Pineal Res 1995; 19:11622.

59. Bryant PA, Trinder J, Curtis N. Sick and tired: does sleep have a vital role in the immune system? Nat Rev Immunol 2004;4: 457-67.

60. Imeri L, Opp MR. How (and why) the immune system makes us sleep. Nat Rev Neurosci 2009;10:199-210.

61. Riemann D, Spiegelhalder K, Feige B, Voderholzer U, Berger M, Perlis M, et al. The hyperarousal model of insomnia: a review of the concept and its evidence. Sleep Med Rev 2010;14:19-31.

62. Parthasarathy S, Vasquez MM, Halonen M, Bootzin R, Quan SF, Martinez FD, et al. Persistent insomnia is associated with mortality risk. Am J Med 2015;128:268-75.

63. Baglioni C, Regen W, Teghen A, Spiegelhalder K, Feige B, Nissen C, et al. Sleep changes in the disorder of insomnia: a meta-analysis of polysomnographic studies. Sleep Med Rev 2014; 18:195-213.

64. Oh DY, Park SM, Choi SW. Daytime neurophysiological hyperarousal in chronic insomnia: a study of qEEG. J Clin Med 2020; 9:3425.

65. Cortoos A, De Valck E, Pattyn N, Mairesse O, Cluydts R. Excitatory versus inhibitory impairments in insomnia patients: an ERP study. Int J Psychophysiol 2014;93:62-9.

66. Bollu PC, Kaur H. Sleep medicine: insomnia and sleep. Mo Med 2019;116:68-75.

67. Javaheri S, Redline S. Insomnia and risk of cardiovascular disease. Chest 2017; 152:435-44.

68. Spiegelhalder K, Scholtes C, Riemann D. The association between insomnia and cardiovascular diseases. Nat Sci Sleep 2010;2:71-8.

69. Hsu CY, Chen YT, Chen MH, Huang CC, Chiang CH, Huang $\mathrm{PH}$, et al. The association between insomnia and increased future cardiovascular events: a nationwide population-based study. Psychosom Med 2015;77:743-51.

70. Sofi F, Cesari F, Casini A, Macchi C, Abbate R, Gensini GF. Insomnia and risk of cardiovascular disease: a meta-analysis. Eur J Prev Cardiol 2014;21:57-64.

71. Hsieh CG, Martin JL. Short sleep, insomnia, and cardiovascular disease. Curr Sleep Med Rep 2019;5:234-42.

72. Lin CL, Chien WC, Chung CH, Wu FL. Risk of type 2 diabetes in patients with insomnia: a population-based historical cohort study. Diabetes Metab Res Rev 2018;34:e2930.

73. Johnson KA, Gordon CJ, Chapman JL, Hoyos CM, Marshall NS, Miller CB, et al. The association of insomnia disorder characterised by objective short sleep duration with hypertension, diabetes and body mass index: a systematic review and meta-analysis. Sleep Med Rev 2021;59:101456.

74. Xia L, Chen GH, Li ZH, Jiang S, Shen J. Alterations in hypothalamus-pituitary-adrenal/thyroid axes and gonadotropin-releasing hormone in the patients with primary insomnia: a clinical research. PLoS One 2013;8:e71065.

75. Crönlein T. Insomnia and obesity. Curr Opin Psychiatry 2016; 29:409-12.

76. Chan WS, Levsen MP, McCrae CS. A meta-analysis of associations between obesity and insomnia diagnosis and symptoms. Sleep Med Rev 2018;40:170-82.

77. Nieters A, Blagitko-Dorfs N, Peter HH, Weber S. Psychophysiological insomnia and respiratory tract infections: results of an infection-diary-based cohort study. Sleep 2019;42:zsz098. 
78. Ruel S, Ivers H, Savard MH, Gouin JP, Lemieux J, Provencher L, et al. Insomnia, immunity, and infections in cancer patients: results from a longitudinal study. Health Psychol 2020;39:35869.

79. Blask DE. Melatonin, sleep disturbance and cancer risk. Sleep Med Rev 2009; 13:257-64.

80. Fang HF, Miao NF, Chen CD, Sithole T, Chung MH. Risk of cancer in patients with insomnia, parasomnia, and obstructive sleep apnea: a nationwide nested case-control study. J Cancer 2015;6:1140-7.

81. Sen A, Opdahl S, Strand LB, Vatten LJ, Laugsand LE, Janszky I. Insomnia and the risk of breast cancer: the HUNT study. Psychosom Med 2017;79:461-8.

82. Shi T, Min M, Sun C, Zhang Y, Liang M, Sun Y. Does insomnia predict a high risk of cancer? A systematic review and meta-analysis of cohort studies. J Sleep Res 2020;29:e12876.

83. Vyazovskiy VV, Delogu A. NREM and REM Sleep: complementary roles in recovery after wakefulness. Neuroscientist 2014;20:203-19.

84. Léger D, Debellemaniere E, Rabat A, Bayon V, Benchenane K,
Chennaoui M. Slow-wave sleep: from the cell to the clinic. Sleep Med Rev 2018;41:113-32.

85. Peever J, Fuller PM. The biology of REM sleep. Curr Biol 2017; 27:R1237-48.

86. Kucharczyk ER, Morgan K, Hall AP. The occupational impact of sleep quality and insomnia symptoms. Sleep Med Rev 2012; 16:547-59.

87. Okajima I, Chen J. The effect of insomnia on changes in anxiety, depression, and social function after a transdiagnostic treatment targeting excessive worry. Sleep Biol Rhythm 2017;15:243-9.

88. Ishak WW, Bagot K, Thomas S, Magakian N, Bedwani D, Larson D, et al. Quality of life in patients suffering from insomnia. Innov Clin Neurosci 2012;9:13-26.

89. Ozminkowski RJ, Wang S, Walsh JK. The direct and indirect costs of untreated insomnia in adults in the United States. Sleep 2007;30:263-73.

90. Matteson-Rusby SE, Pigeon WR, Gehrman P, Perlis ML. Why treat insomnia? Prim Care Companion J Clin Psychiatry 2010; 12:PCC.08r00743. 